\title{
Synthesis of Highly Functionalized Fluorinated Cispentacin Derivatives
}

by Melinda Nonn $\left.{ }^{a}\right)^{b}$, , Loránd Kiss $\left.{ }^{a}\right)$, Mikko M. Hänninen ${ }^{c}$ ), Reijo Sillanpääc ${ }^{c}$, and Ferenc Fülöp*a $)^{b}$ )

a) Institute of Pharmaceutical Chemistry, University of Szeged, HU-6720, Eötvös 6

b) Stereochemistry Research Group of the Hungarian Academy of Sciences, University of Szeged, HU-6720 Szeged, Eötvös 6

c) Department of Chemistry, University of Jyväskylä, FI-40014, Jyväskylä

Dedicated to Professor Dieter Seebach on the occasion of his 75 th birthday

Fluorinated highly functionalized cispentacin derivatives were synthetised starting from an unsaturated bicyclic $\beta$-lactam through $\mathrm{C}=\mathrm{C}$ bond functionalization via the dipolar cycloaddition of a nitrile oxide, isoxazoline opening, and fluorination by $\mathrm{OH} / \mathrm{F}$ exchange.

Introduction. - As a result of their biological potential, cyclic $\beta$-amino acids are of considerable importance in medicinal chemistry. As conformationally restricted derivatives, they are building blocks for the construction of biologically active peptides [1][2]. They include cispentacin (1), an important potent antifungal [1a][1b]. Multisubstituted aminocyclopentanecarboxylic acids such as Peramivir (2) and related analogs $3\left(\mathrm{R}^{1}=\mathrm{H}, \mathrm{OH} ; \mathrm{R}^{2}=\mathrm{Et}, \mathrm{Pr}\right)$ exhibit strong antiviral properties [3].

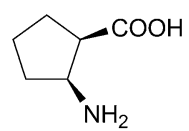

1<smiles>CCC(CC)C(NC(C)(C)C)[C@H]1[C@H](O)[C@@H](C(=O)O)C[C@H]1NC(=N)N</smiles>

2<smiles>[R]C([R])[C@H](N)[C@@H]1C([R])[C@@H](C(=O)O)C[C@@H]1NC(=N)N</smiles>

3

Fluorinated $\alpha$ - and acyclic $\beta$-amino acids comprise an expanding area of research, with increasing impact in both chemistry and biochemistry. They are valuable in medicinal chemistry as enzyme inhibitors, antitumour agents, or antibiotics [4][5]. Only a small number of fluorinated cyclic $\beta$-amino acids have been prepared so far, this being particularly true for the five-membered derivatives [6].

We recently reported the synthesis of highly functionalized cispentacin stereoisomers 7 and 8 from bicyclic $\beta$-lactam 4 by means of the regio- and stereoselective 1,3dipolar cycloaddition of a nitrile oxide (acetonitrile $\mathrm{N}$-oxide) to ethyl cis-and trans-2aminocyclopentene-3-carboxylates, followed by the stereoselective opening of the isoxazoline ring (Scheme 1) [7].

Results and Discussion. - Our current aim was to synthesize highly functionalized regio- and stereoisomers of F-containing five-membered cyclic $\beta$-amino acid deriva- 


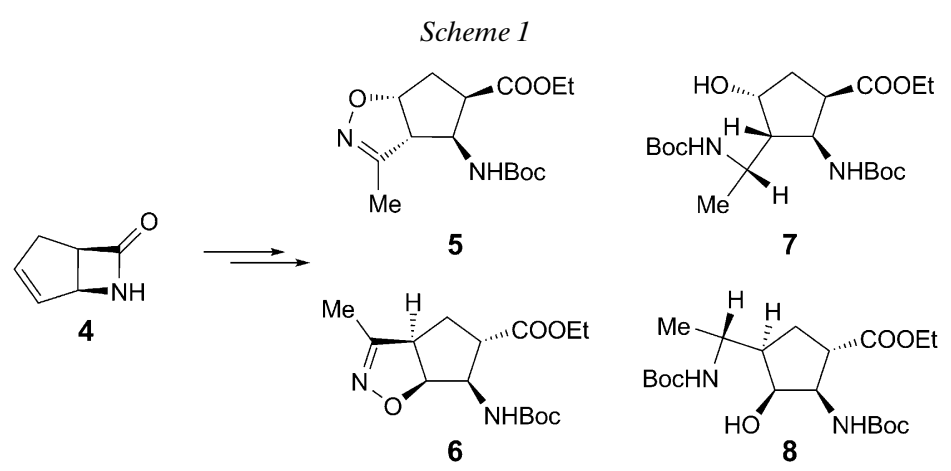

tives from bicyclic $\beta$-lactam 4 through selective transformation of its $\mathrm{C}=\mathrm{C}$ bond by the dipolar cycloaddition of a nitrile oxide, followed by reductive isoxazoline opening and $\mathrm{H} / \mathrm{F}$ exchange.

Accordingly, novel $\mathrm{OH}$-containing, multifunctionalized $\beta$-aminocyclopentanecarboxylates were prepared by reductive ring opening of the isoxazoline skeleton of 9 and 10 [8] (Scheme 2). In contrast to our earlier experiments on Me-substituted compounds (cf. Scheme 1) [7], the reductive isoxazoline opening of Et-substituted cis- and transisoxazoline-fused derivatives 9 and $\mathbf{1 0}$ under similar experimental conditions, with $\mathrm{NaBH}_{4}$ in the presence of $\mathrm{NiCl}_{2}$ in $\mathrm{EtOH} / \mathrm{THF}$, did not prove to be $100 \%$ stereoselective. Both transformations furnished two diastereoisomers, 11 (Fig. 1) and 12, or $\mathbf{1 3}$ and 14, in a ratio of $3: 1$, the major products, $\mathbf{1 1}$ and $\mathbf{1 3}$, respectively, resulting from $\mathrm{H}$ attack on the isoxazoline from the same face of the carbamate (Scheme 2; for several related transformations, see [3]). The products $\mathbf{1 1}+\mathbf{1 2}$ and $\mathbf{1 3}+\mathbf{1 4}$ were separated and isolated by column chromatography on $\mathrm{SiO}_{2}$.
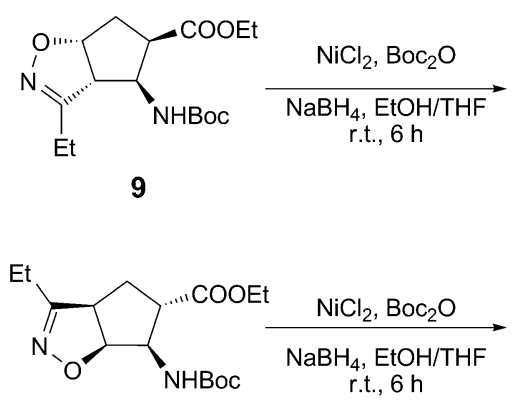

10

Scheme 2

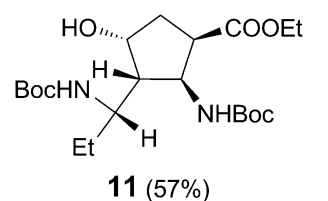

$11(57 \%)$

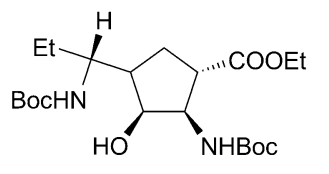

$13(64 \%)$

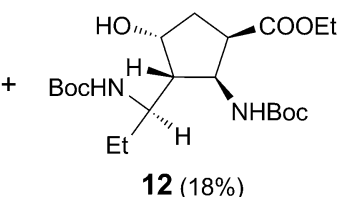

$12(18 \%)$

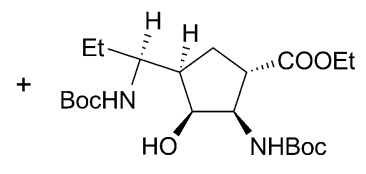

$14(21 \%)$

New multifunctionalized hydroxylated cispentacin analogs containing a longer alkyl chain were next prepared by cycloaddition of the nitrile oxide formed from 2ethylbutyraldehyde oxime in the presence of $N$-chlorosuccinimide (NCS; Huisgen's conditions) to ethyl cis- and trans-2-aminocyclohexenecarboxylates, $\mathbf{1 5}$ and $\mathbf{1 8}$, 


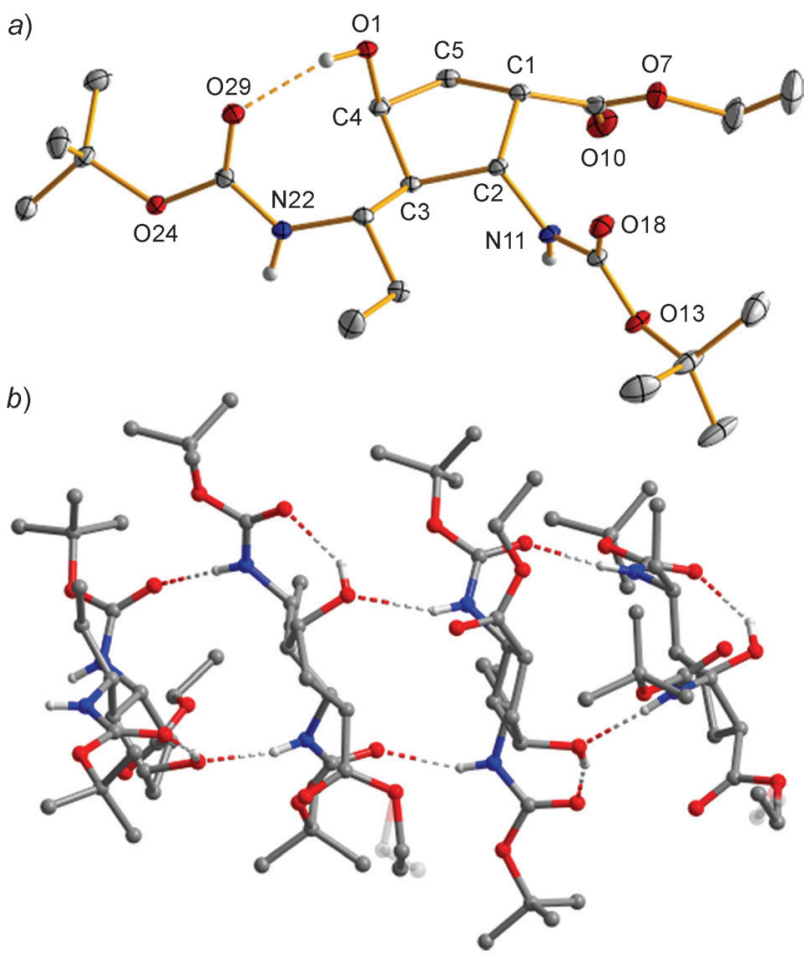

Fig. 1. a) Molecular structure of compound 11. Only one of two similar molecules in the asymmetric unit is presented. b) Ball-and-stick model of $\mathbf{1 1}$ showing inter-and intramolecular H-bonds. Thermal ellipsoids have been drawn at $30 \%$ probability level, and the $\mathrm{C}-\mathrm{H} \mathrm{H}$-atoms are omitted for clarity.

respectively (Scheme 3). The cycloaddition to $\mathbf{1 5}$ gave isoxazoline-fused amino ester regioisomers $\mathbf{1 6 / 1 7}$ in a ratio of $7.5: 1$ (Scheme 3), the major product containing the Oatom of the isoxazoline skeleton farther from the carbamate group (for analogous transformations, see [8a]). The products were separated by chromatography. Similarly as with other nitrile oxides [8b], the cycloaddition to the trans counterpart 18 selectively afforded only cycloadduct 19 (Scheme 3$)$.

Analogously to $\mathbf{9}$ and $\mathbf{1 0}$, the reductive ring openings of isoxazoline-fused cis- and trans-amino esters 16 and $\mathbf{1 9}$, respectively, with $\mathrm{NaBH}_{4} / \mathrm{NiCl}_{2}$ each furnished two products, 20 (Fig. 2; the $\mathrm{H}_{2} \mathrm{O}$ adduct of the compound)/21, or 22/23, respectively, in a ratio of $2: 1$ and $3: 1$, which were separated by column chromatography (Scheme 4).

Introduction of a F-atom in the skeleton of the major isomers of the synthesized highly-functionalized cispentacin derivatives possessing a $\mathrm{OH}$ substituent was achieved through $\mathrm{H} / \mathrm{F}$ exchange with Deoxo-Fluor ${ }^{\circledR}$ (= bis(2-methoxyethyl)aminosulfur trifluoride) as reagent.

Fluorination of 7, 11, and $\mathbf{2 0}$ in dry toluene at $0^{\circ}$ for $2 \mathrm{~h}$ afforded the corresponding fluorinated compounds with inversion, $24 \mathbf{a}-\mathbf{2 4} \mathbf{c}$, and the elimination products $\mathbf{2 5 a}-\mathbf{2 5 c}$, respectively (for analogous experimental results, see [6a][6b] and ref. cit. therein; Scheme 5), which were separated by column chromatography. No experimental 


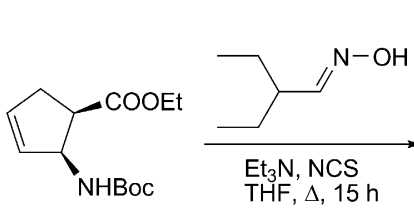

15

Scheme 3

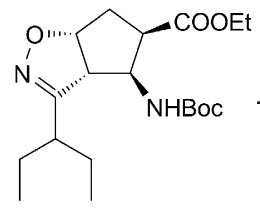

$16(45 \%)$

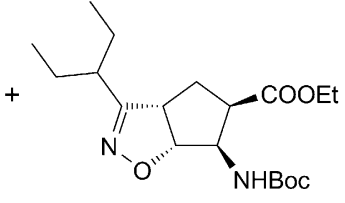

$17(6 \%)$
NaOEt, EtOH

18

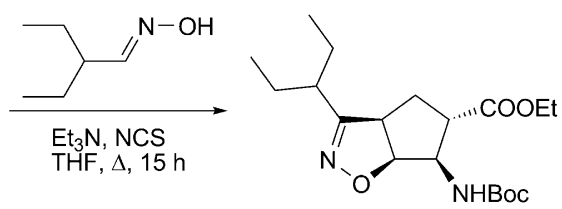

$19(50 \%)$

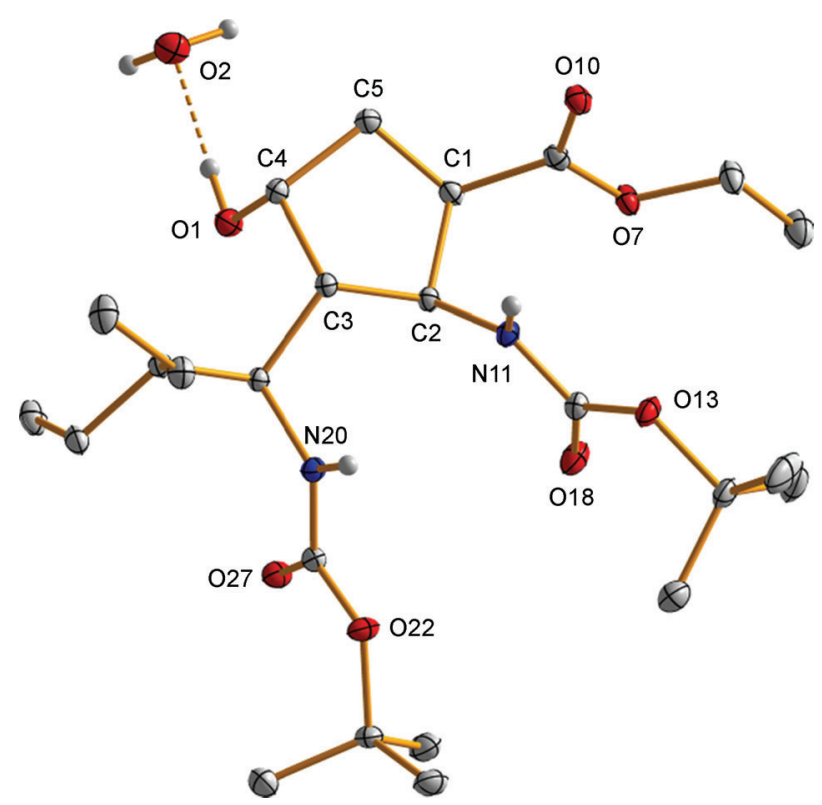

Fig. 2. Molecular structure of compound 20 $\cdot \mathrm{H}_{2} \mathrm{O}$. Thermal ellipsoids have been drawn at $30 \%$ probability level, and the $\mathrm{C}-\mathrm{H} \mathrm{H}$-atoms are omitted for clarity.

conditions were found under which the large amounts of elimination products could be avoided.

Under similar conditions, fluorination of the trans counterparts $8, \mathbf{1 3}$, and $\mathbf{2 2}$ provided the required fluorinated products $\mathbf{2 6 a}$ and $\mathbf{2 6 b}$, unfortunately again together with large quantities of elimination products $\mathbf{2 7 a - 2 7 c}$, respectively (Scheme 6). 
Scheme 4<smiles>CCOC(=O)C1CC2ON=C(C(CC)CC)C2C1NC(=O)OCc1ccccc1</smiles>

16

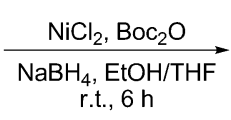

r.t., $6 \mathrm{~h}$

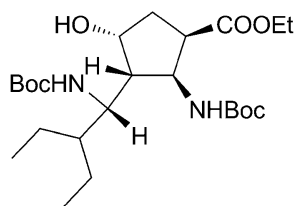

$20(53 \%)$

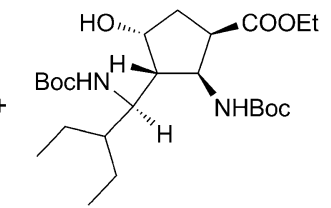

$21(27 \%)$

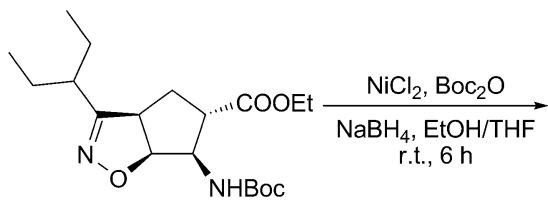

19

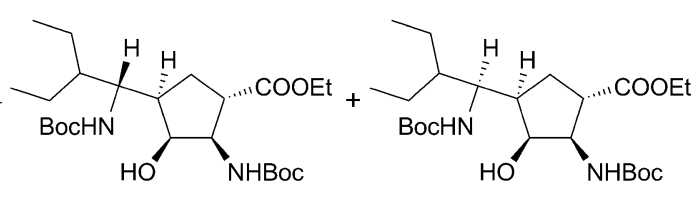

$23(20 \%)$

Scheme 5
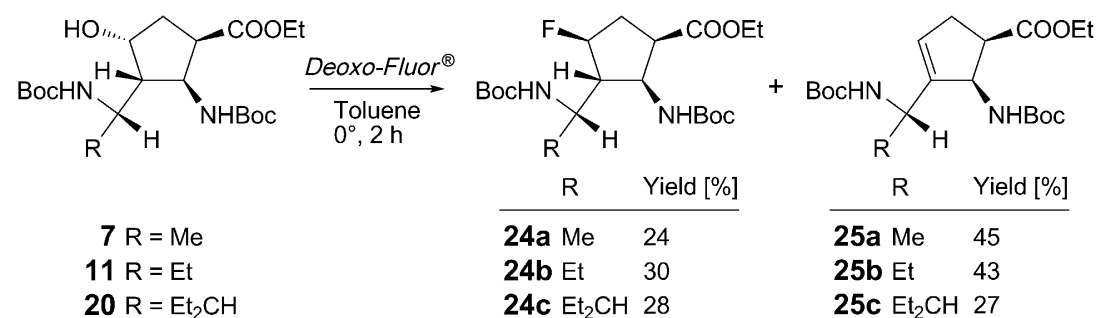

$11 \mathrm{R}=\mathrm{Et}$

$20 \mathrm{R}=\mathrm{Et}_{2} \mathrm{CH}$

24c $\mathrm{Et}_{2} \mathrm{CH} 28$

25c $\mathrm{Et}_{2} \mathrm{CH} 27$

Scheme 6

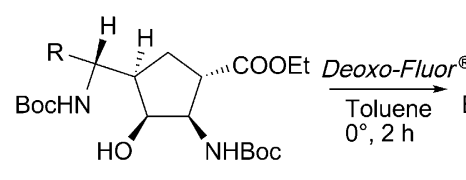

(n)

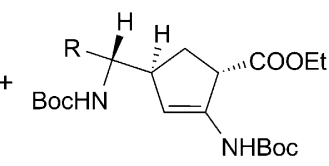

\begin{tabular}{ll}
\multicolumn{1}{c}{$\mathrm{R}$} & Yield [\%] \\
\hline 26a Me & 26 \\
26b Et & 15 \\
26c $\mathrm{Et}_{2} \mathrm{CH}$ & 0
\end{tabular}

\begin{tabular}{ll}
\multicolumn{1}{c}{$\mathrm{R}$} & Yield [\%] \\
\hline 27a $\mathrm{Me}$ & 48 \\
27b Et & 39 \\
27c $\mathrm{Et}_{2} \mathrm{CH}$ & 58
\end{tabular}

To summarize, highly functionalized fluorinated $\beta$-aminocyclopentanecarboxylate regio- and stereoisomers containing multiple stereogenic centers were synthesized from $\beta$-aminocyclopentenecarboxylates through the 1,3-dipolar cycloaddition of nitrile oxides and reductive ring opening of the isoxazoline skeleton, followed by $\mathrm{H} / \mathrm{F}$ exchange. These products may be regarded not only as fluorinated cispentacin derivatives, but as precursors for the preparation of $\beta$-amino acid-modified peramivir analogs.

We are grateful to the Hungarian Research Foundation (OTKA No. NK81371 and K100530) for financial support. 


\section{Experimental Part}

General. The chemicals were purchased from Aldrich. The solvents were used as received from the supplier. M.p.: Kofler apparatus. NMR Spectra: Bruker DRX 400 spectrometer, chemical shifts, $\delta$, in ppm rel. to TMS as internal standard, with $\mathrm{CDCl}_{3}$ as solvent. MS: Finnigan MAT $95 S$ spectrometer. Elemental analyses: Perkin-Elmer CHNS-2400 Ser II elemental analyzer.

Synthesis of 2-Ethylbutyraldehyde Oxime (=(1E)-2-Ethyl-N-hydroxybutan-1-imine). To a soln. of 2ethylbutyraldehyde (=2-ethylbutanal; $50 \mathrm{mmol})$ in EtOH $(50 \mathrm{ml})$, dry pyridine $(150 \mathrm{mmol})$, and $\mathrm{NH}_{2} \mathrm{OH} \cdot \mathrm{HCl}(50 \mathrm{mmol})$ were added, and the mixture was stirred under reflux for $7 \mathrm{~h}$. The mixture was then concentrated under reduced pressure, and the crude residue was purified by column chromatography (CC) on $\mathrm{SiO}_{2}$ (hexane/AcOEt) to give (1E)-2-ethyl- $N$-hydroxybutan-1-imine.

General Procedure for the Synthesis of Isoxazoline-Fused $\beta$-Aminocyclopentanecarboxylates. To a soln. of amino ester $\mathbf{1 5}$ or $18(19.6 \mathrm{mmol})$ in THF $(70 \mathrm{ml}),(1 E)$-2-ethyl- $N$-hydroxybutan-1-imine $(118 \mathrm{mmol}), \mathrm{Et}_{2} \mathrm{NH}(19.6 \mathrm{mmol})$, and $N$-chlorosuccinimide (=1-chloropyrrolidine-2,5-dione; $78.4 \mathrm{mmol}$ ) were added, and the mixture was stirred at r.t. for $48 \mathrm{~h}$. The mixture was then diluted with AcOEt $(75 \mathrm{ml})$, washed with $\mathrm{H}_{2} \mathrm{O}(3 \times 20 \mathrm{ml})$, dried $\left(\mathrm{Na}_{2} \mathrm{SO}_{4}\right)$, and concentrated under reduced pressure. The crude mixture was purified by $\mathrm{CC}\left(\mathrm{SiO}_{2}\right.$; hexane/AcOEt to give 16/17 and 19).

General Procedure for Isoxazoline Ring Opening. To a soln. of dihydroisoxazol 9, 10, 16, or 19 $(1.46 \mathrm{mmol})$ in $10 \mathrm{ml}$ of EtOH/THF $3: 1(\mathrm{v} / \mathrm{v}), \mathrm{NiCl}_{2}(2.92 \mathrm{mmol})$ and $\mathrm{Boc}_{2} \mathrm{O}(2.92 \mathrm{mmol})$ were added. After stirring for $10 \mathrm{~min}, \mathrm{NaBH}_{4}(2.92 \mathrm{mmol})$ was added in portions. The mixture was stirred at r.t. for $5 \mathrm{~h}$, and the reaction was then quenched by the addition of $\mathrm{H}_{2} \mathrm{O}(5 \mathrm{ml})$. The mixture was filtered through Celite pad, and the filtrate was concentrated under reduced pressure. The residue was diluted with AcOEt $(30 \mathrm{ml})$, washed with $\mathrm{H}_{2} \mathrm{O}(3 \times 10 \mathrm{ml})$, dried $\left(\mathrm{Na}_{2} \mathrm{SO}_{4}\right)$, and evaporated in vacuo. The products 11/12, 13/14, 20/21, and 22/23 were purified and separated by $\mathrm{CC}\left(\mathrm{SiO}_{2}\right.$; hexane/AcOEt $)$.

General Procedure for the Synthesis of F-Containing $\beta$-Aminocyclopentanecarboxylates. To a soln. of hydroxy compounds 7, 8, 11, 13, 20, or $22(0.5 \mathrm{mmol})$ in dry toluene $(10 \mathrm{ml})$, Deoxo-Fluor ${ }^{\circledR}$ soln. $(50 \%$ in toluene, $0.6 \mathrm{mmol}$ ) was added at $0^{\circ}$ under Ar. The mixture was stirred at $0^{\circ}$ for $2 \mathrm{~h}$, and the mixture was then diluted with AcOEt, washed with sat. $\mathrm{NaHCO}_{3}$ soln. $(3 \times 10 \mathrm{ml})$, followed by $\mathrm{H}_{2} \mathrm{O}(2 \times 10 \mathrm{ml})$, dried $\left(\mathrm{Na}_{2} \mathrm{SO}_{4}\right)$, and concentrated under reduced pressure. The crude residue was purified by $\mathrm{CC}\left(\mathrm{SiO}_{2}\right.$; hexane/AcOEt) to furnish $24 \mathbf{a}-24 \mathbf{c}, 25 \mathbf{a}-25 \mathbf{c}, 26 \mathbf{a}$ and $26 \mathbf{b}$, and $27 \mathbf{a}-\mathbf{2 7} \mathbf{c}$.

Ethyl (1R*,2S*,3S*,4R*)-2-\{[(tert-Butoxy)carbonyl]amino $\}-3-\left(\left(1 \mathrm{~S}^{*}\right)-1-\{[\right.$ (tert-butoxy) carbonyl]amino/propyl)-4-hydroxycyclopentanecarboxylate $(\mathbf{1 1})$. White solid. Yield: $57 \% . R_{\mathrm{f}}$ (hexane/AcOEt) 0.24. M.p. 95-96 ${ }^{\circ}{ }^{1} \mathrm{H}-\mathrm{NMR}\left(400 \mathrm{MHz}, \mathrm{CDCl}_{3}\right): 0.95(t, J=7.36, \mathrm{Me}) ; 1.30(t, J=7.2, \mathrm{Me}) ; 1.45\left(s,{ }^{\mathrm{B} u}\right)$; $1.49\left(s,{ }^{t} \mathrm{Bu}\right) ; 1.94-2.16\left(\mathrm{~m}, 2 \mathrm{CH}_{2}\right) ; 3.34-3.65(m, \mathrm{H}-\mathrm{C}(1)) ; 3.79-3.89(m, \mathrm{H}-\mathrm{C}(3)) ; 4.12-4.30(m$, $\left.\mathrm{H}-\mathrm{C}(2), \mathrm{CH}, \mathrm{CH}_{2} \mathrm{O}\right) ; 4.44-4.55$ ( $\left.m, \mathrm{H}-\mathrm{C}(4)\right) ; 5.26-5.37$ (br. $\left.s, \mathrm{NH}\right) ; 5.61-5.72$ (br. $\left.s, \mathrm{NH}\right) .{ }^{13} \mathrm{C}-\mathrm{NMR}$ $\left(100 \mathrm{MHz}, \mathrm{CDCl}_{3}\right): 10.9 ; 13.7 ; 27.9 ; 28.0 ; 28.4 ; 36.4 ; 43.8 ; 50.1 ; 50.3 ; 53.8 ; 60.3 ; 72.9 ; 79.3 ; 79.6 ; 155.2$; 157.3; 174.9. ESI-MS: $431\left([M+1]^{+}\right)$. Anal. calc. for $\mathrm{C}_{21} \mathrm{H}_{38} \mathrm{~N}_{2} \mathrm{O}_{7}$ : C 58.58, H 8.90, N 6.51; found: C 58.55, H 8.92, N 6.49.

Ethyl $\left(1 \mathrm{R}^{*}, 2 \mathrm{~S} *, 3 \mathrm{~S}^{*}, 4 \mathrm{R} *\right)-2-\{[($ tert-Butoxy $)$ carbonyl]amino $\}-3-((1 \mathrm{R} *)-1-\{[($ tert-butoxy $)$ carbonyl $\}-$ aminolpropyl)-4-hydroxycyclopentanecarboxylate (12). White solid. Yield:18\%. $R_{\mathrm{f}}$ (hexane/AcOEt $2: 1)$ 0.33. M.p. $125-127^{\circ} .{ }^{1} \mathrm{H}-\mathrm{NMR}\left(400 \mathrm{MHz}, \mathrm{CDCl}_{3}\right): 2.97(t, J=7.2, \mathrm{Me}) ; 1.28(t, J=7.1, \mathrm{Me}) ; 1.43(s$, $\left.{ }^{t} \mathrm{Bu}\right) ; 1.48\left(s,{ }^{t} \mathrm{Bu}\right) ; 1.65-1.89\left(m, \mathrm{CH}_{2}\right) ; 1.96-2.18\left(m, \mathrm{CH}_{2}\right) ; 3.50-3.61(m, \mathrm{H}-\mathrm{C}(1)) ; 3.66-3.77(m$, $\mathrm{H}-\mathrm{C}(3))$; 4.06-4.27 ( $\left.m, \mathrm{CH}, \mathrm{H}-\mathrm{C}(2) ; \mathrm{CH}_{2} \mathrm{O}\right) ; 4.38-4.49(m, \mathrm{H}-\mathrm{C}(4)) ; 4.58-4.72$ (br. $\left.s, 2 \mathrm{NH}\right)$. ${ }^{13} \mathrm{C}-\mathrm{NMR}\left(100 \mathrm{MHz}, \mathrm{CDCl}_{3}\right): 10.0 ; 13.8 ; 25.3 ; 27.9 ; 35.4 ; 45.6 ; 50.9 ; 51.9 ; 56.6 ; 60.2 ; 71.3 ; 78.9 ; 79.5$; 154.4; 156.7; 174.1. ESI-MS: $431\left([M+1]^{+}\right)$. Anal. calc. for $\mathrm{C}_{21} \mathrm{H}_{38} \mathrm{~N}_{2} \mathrm{O}_{7}: \mathrm{C} 58.58, \mathrm{H} 8.90, \mathrm{~N} 6.51$; found: $\mathrm{C}$ $59.12, \mathrm{H} 8.02, \mathrm{~N} 6.60$.

Ethyl (1R*,2S*,3R*,4S*)-2-\{[(tert-Butoxy)carbonyl]amino\}-4-((1S*)-1-\{[(tert-butoxy) carbonyl]amino/propyl)-3-hydroxycyclopentanecarboxylate (13). White solid. Yield: $64 \%$. $R_{\mathrm{f}}$ (hexane/AcOEt) 0.57. M.p. 216-217 ${ }^{\circ}{ }^{1} \mathrm{H}-\mathrm{NMR}\left(400 \mathrm{MHz}, \mathrm{CDCl}_{3}\right): 0.96(t, J=7.4, \mathrm{Me}) ; 1.25-1.33$ ( $\left.m, \mathrm{Me}\right) ; 1.44\left(\mathrm{~s},{ }^{t} \mathrm{Bu}\right)$; $1.49\left(s,{ }^{t} \mathrm{Bu}\right) ; 1.76-2.38\left(m, 2 \mathrm{CH}_{2}\right) ; 2.47-2.62(m, \mathrm{H}-\mathrm{C}(1)) ; 2.76-2.96(m, \mathrm{H}-\mathrm{C}(4)) ; 3.10-3.19(m$, $\mathrm{H}-\mathrm{C}(2)) ; 3.68-3.80(m, \mathrm{CH}) ; 4.10-4.24\left(m, \mathrm{CH}_{2} \mathrm{O}\right) ; 4.28-4.38$ (br. $\left.s, \mathrm{NH}\right) ; 4.42-4.47(m, \mathrm{H}-\mathrm{C}(3))$; 5.01-5.25 (br. $s, \mathrm{NH}) .{ }^{13} \mathrm{C}-\mathrm{NMR}\left(100 \mathrm{MHz}, \mathrm{CDCl}_{3}\right): 10.3 ; 11.1 ; 27.0 ; 28.1 ; 32.4 ; 33.2 ; 45.2 ; 52.3 ; 50.8 ; 54.1$; 
58.8; 72.1; 77.0; 154.5; 156.5; 175.4. ESI-MS: $431\left([M+1]^{+}\right)$. Anal. calc. for $\mathrm{C}_{21} \mathrm{H}_{38} \mathrm{~N}_{2} \mathrm{O}_{7}: \mathrm{C} 58.58, \mathrm{H}$ 8.90, N 6.51; found: C 58.57, H 8.91, N 6.49.

Ethyl $\left(1 \mathrm{R}^{*}, 2 \mathrm{~S} *, 3 \mathrm{R}^{*}, 4 \mathrm{~S}^{*}\right)-2-\{[($ tert-Butoxy $)$ carbonyl]amino $\}-4-((1 \mathrm{R} *)-1-\{[($ tert-butoxy $)$ carbonyl $]-$ amino\}propyl)-3-hydroxycyclopentanecarboxylate (14). Brownish oil. Yield: $21 \% . R_{\mathrm{f}}$ (hexane/AcOEt) 0.35. ${ }^{1} \mathrm{H}-\mathrm{NMR}\left(400 \mathrm{MHz}, \mathrm{CDCl}_{3}\right): 0.96(t, J=7.6, \mathrm{Me}) ; 1.25-1.33(\mathrm{~m}, \mathrm{Me}) ; 1.45\left(s,{ }^{t} \mathrm{Bu}\right) ; 1.50\left(s,{ }^{t} \mathrm{Bu}\right)$; $1.81-2.15\left(m, 2 \mathrm{CH}_{2}\right) ; 2.24-2.36(m, \mathrm{H}-\mathrm{C}(4)) ; 2.74-2.86(m, \mathrm{H}-\mathrm{C}(1)) ; 3.69-3.82(m, \mathrm{CH}) ; 4.09-4.26$ $\left(m, \mathrm{H}-\mathrm{C}(2), \mathrm{H}-\mathrm{C}(3), \mathrm{CH}_{2} \mathrm{O}\right) ; 4.29-4.39$ (br. $\left.s, \mathrm{NH}\right) ; 5.03-5.20$ (br. $\left.s, \mathrm{NH}\right) .{ }^{13} \mathrm{C}-\mathrm{NMR}(100 \mathrm{MHz}$, $\left.\mathrm{CDCl}_{3}\right): 10.0 ; 11.0 ; 27.9 ; 28.0 ; 31.1 ; 35.5 ; 45.2 ; 51.7 ; 51.1 ; 56.5 ; 60.1 ; 71.6 ; 78.1 ; 154.4 ; 155.5 ; 174.6$. ESI-MS: $453\left([M+\mathrm{Na}]^{+}\right)$. Anal. calc. for $\mathrm{C}_{21} \mathrm{H}_{38} \mathrm{~N}_{2} \mathrm{O}_{7}: \mathrm{C} 58.58, \mathrm{H}$ 8.90, N 6.51; found: C 58.57, H 8.98, N 6.50.

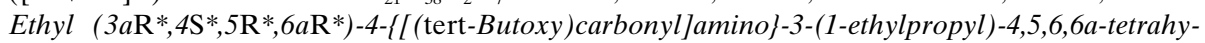
dro-3aH-cyclopenta[d] [1,2]oxazole-5-carboxylate (16). Brownish oil. Yield: $45 \%$. $R_{\mathrm{f}}$ (hexane/AcOEt) 0.72. ${ }^{1} \mathrm{H}$-NMR (400 MHz, $\left.\mathrm{CDCl}_{3}\right): 0.90-1.00(m, 2 \mathrm{Me}) ; 1.30(t, J=7.1, \mathrm{Me}) ; 1.47\left(\mathrm{~s},{ }^{t} \mathrm{Bu}\right) ; 1.54-1.84(\mathrm{~m}$, $\left.2 \mathrm{CH}_{2}\right) ; 2.25-2.41\left(m, \mathrm{CH}_{2}\right) ; 2.46-2.55(m, \mathrm{H}-\mathrm{C}(5)) ; 2.94-3.07(m, \mathrm{H}-\mathrm{C}(3 \mathrm{a})) ; 3.64-3.75(m, \mathrm{CH})$; 4.16-4.31 ( $\left.m, \mathrm{CH}_{2} \mathrm{O}, \mathrm{H}-\mathrm{C}(4)\right) ; 5.06-5.20(m, \mathrm{H}-\mathrm{C}(6 \mathrm{a}), \mathrm{NH}) .{ }^{13} \mathrm{C}-\mathrm{NMR}\left(100 \mathrm{MHz}, \mathrm{CDCl}_{3}\right): 11.4 ; 12.5$; $14.5 ; 24.4 ; 25.9 ; 28.6 ; 31.6 ; 36.9 ; 41.0 ; 45.6 ; 52.1 ; 57.1 ; 61.6 ; 62.9 ; 83.7 ; 155.4 ; 160.8$. ESI-MS: $369\left([M+1]^{+}\right)$. Anal. calc. for $\mathrm{C}_{19} \mathrm{H}_{32} \mathrm{~N}_{2} \mathrm{O}_{5}$ : C 61.93, H 8.75, N 7.60; found: C 61.92, H 8.76, N 6.7.58.

Ethyl $\left(3 a \mathrm{R} *, 5 \mathrm{~S}^{*}, 6 \mathrm{~S} *, 6 a \mathrm{R} *\right)-6-\{[$ (tert-Butoxy) carbonyl]amino\}-3-(1-ethylpropyl)-4,5,6,6a-tetrahydro-3aH-cyclopenta[d] [1,2]oxazole-5-carboxylate (17). Yellowish oil. Yield: $6 \%$. $R_{\mathrm{f}}$ (hexane/AcOEt) 0.52. ${ }^{1} \mathrm{H}-\mathrm{NMR}\left(400 \mathrm{MHz}, \mathrm{CDCl}_{3}\right): 1.21-1.33(\mathrm{~m}, 3 \mathrm{Me}) ; 1.46\left(s,{ }^{ } \mathrm{Bu}\right) ; 1.51-2.20\left(m, 2 \mathrm{CH}_{2}\right) ; 2.32-2.46$ $\left(m, \mathrm{CH}_{2}\right) ; 2.93-3.07(m, \mathrm{H}-\mathrm{C}(5)) ; 3.44-3.54(m, \mathrm{H}-\mathrm{C}(3 \mathrm{a})) ; 4.01-4.24\left(m, \mathrm{CH}, \mathrm{CH}_{2} \mathrm{O}\right) ; 4.28-4.40(m$, $\mathrm{H}-\mathrm{C}(6)) ; 4.87-4.92(m, \mathrm{H}-\mathrm{C}(6 \mathrm{a})) ; 5.64-5.80$ (br. $s, \mathrm{NH}) .{ }^{13} \mathrm{C}-\mathrm{NMR}\left(100 \mathrm{MHz}, \mathrm{CDCl}_{3}\right): 10.5 ; 11.2 ; 14.5$; $23.9 ; 26.2 ; 30.0 ; 31.4 ; 35.9 ; 42.1 ; 44.9 ; 52.2 ; 56.9 ; 59.6 ; 62.9 ; 85.0 ; 155.4 ; 161.4$. ESI-MS: $369\left([M+1]^{+}\right)$. Anal. calc. for $\mathrm{C}_{19} \mathrm{H}_{32} \mathrm{~N}_{2} \mathrm{O}_{5}$ : C 61.93, H 8.75, N 7.60; found: C 61.91, H 8.76, N 7.61.

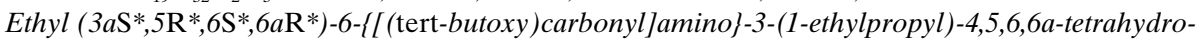
$3 a \mathrm{H}$-cyclopenta[d] [1,2] oxazole-5-carboxylate $(\mathbf{1 9})$. White solid. Yield: $50 \% . R_{\mathrm{f}}$ (hexane/AcOEt) 0.38 . M.p. 95-96 $.{ }^{1} \mathrm{H}-\mathrm{NMR}\left(400 \mathrm{MHz}, \mathrm{CDCl}_{3}\right)$ : $0.89-1.00(m, 2 \mathrm{Me}) ; 1.29(t, J=7.1, \mathrm{Me}) ; 1.46\left(s,{ }^{t} \mathrm{Bu}\right) ; 1.55-$ $1.74\left(m, 2 \mathrm{CH}_{2}\right) ; 1.97-2.04\left(m, 1 \mathrm{H}\right.$ of $\left.\mathrm{CH}_{2}\right) ; 2.14-2.31\left(m, 1 \mathrm{H}\right.$ of $\left.\mathrm{CH}_{2}, \mathrm{H}-\mathrm{C}(5)\right) ; 2.38-2.49(m$, $\mathrm{H}-\mathrm{C}(3 \mathrm{a})) ; 3.62-3.73(m, \mathrm{CH}) ; 4.09-4.34\left(m, \mathrm{H}-\mathrm{C}(6), \mathrm{CH}_{2} \mathrm{O}\right) ; 4.85-4.91(m, \mathrm{H}-\mathrm{C}(6 \mathrm{a})) ; 5.19-5.26$ (br. $s, \mathrm{NH}) .{ }^{13} \mathrm{C}-\mathrm{NMR}\left(100 \mathrm{MHz}, \mathrm{CDCl}_{3}\right): 10.5 ; 11.6 ; 13.6 ; 23.6 ; 25.3 ; 27.8 ; 29.9 ; 39.8 ; 46.4 ; 50.9 ; 59.5 ; 60.6$; $79.0 ; 82.5 ; 154.6 ; 162.4 ; 172.2$. ESI-MS: $369\left([M+1]^{+}\right)$. Anal. calc. for $\mathrm{C}_{19} \mathrm{H}_{32} \mathrm{~N}_{2} \mathrm{O}_{5}: \mathrm{C} 61.93, \mathrm{H} 8.75, \mathrm{~N}$ 7.60; found: C 61.95, H 8.74, N 7.59.

Ethyl $\left(1 \mathrm{R}^{*}, 2 \mathrm{~S}^{*}, 3 \mathrm{~S}^{*}, 4 \mathrm{R}^{*}\right)-2-\left\{[\right.$ (tert-Butoxy) carbonyl]amino $\}-3-\left(\left(1 \mathrm{~S}^{*}\right)-1-\{[\right.$ (tert-butoxy) carbonyl]amino\}-2-ethylbutyl)-4-hydroxycyclopentanecarboxylate (20). White solid. Yield: $53 \% . R_{\mathrm{f}}$ (hexane/ AcOEt) 0.26. M.p. $99-100^{\circ}$. ${ }^{1} \mathrm{H}-\mathrm{NMR}\left(400 \mathrm{MHz}, \mathrm{CDCl}_{3}\right): 0.80-0.93(m, 2 \mathrm{Me}) ; 1.24-1.67\left(m, 2^{t} \mathrm{Bu}\right.$, $\left.\mathrm{CH}_{2}\right) ; 1.97-2.21\left(m, 1 \mathrm{H}\right.$ of $\left.\mathrm{CH}_{2}, \mathrm{H}-\mathrm{C}(1), \mathrm{H}-\mathrm{C}(3)\right) ; 3.31-3.40(m, \mathrm{CH}) ; 3.77-3.89(m, \mathrm{CH}) ; 4.12-4.28$ $\left(m, \mathrm{H}-\mathrm{C}(2), \mathrm{CH}_{2} \mathrm{O}\right) ; 4.40-4.52(m, \mathrm{H}-\mathrm{C}(4)) ; 5.28-5.38$ (br. $\left.s, \mathrm{NH}\right) ; 5.80-5.90$ (br. $\left.s, \mathrm{NH}\right) .{ }^{13} \mathrm{C}-\mathrm{NMR}$ $\left(100 \mathrm{MHz}, \mathrm{CDCl}_{3}\right)$ : 9.6; 9.9; $13.7 ; 20.8 ; 21.4 ; 27.9 ; 28.0 ; 36.5 ; 43.0 ; 43.66 ; 50.1 ; 50.2 ; 50.4 ; 60.4 ; 73.4 ; 79.2$; $79.5 ; 155.1 ; 157.2 ; 175.1$. ESI-MS: $495\left([M+\mathrm{Na}]^{+}\right)$. Anal. calc. for $\mathrm{C}_{24} \mathrm{H}_{44} \mathrm{~N}_{2} \mathrm{O}_{7}: \mathrm{C} 60.99, \mathrm{H} 9.38, \mathrm{~N} 5.93$; found: C 61.01, H, 9.39, N, 5.94 .

Ethyl $\left(1 \mathrm{R}^{*}, 2 \mathrm{~S}^{*}, 3 \mathrm{~S}^{*}, 4 \mathrm{R}^{*}\right)-2-\left\{\left[\left(\right.\right.\right.$ tert-Butoxy) carbonyl]amino\}-3-((1, $\left.\mathrm{R}^{*}\right)-1-\{[($ tert-butoxy) carbonyl]amino\}-2-ethylbutyl)-4-hydroxycyclopentanecarboxylate (21). White solid. Yield: $27 \%$. $R_{\mathrm{f}}$ (hexane/ AcOEt) 0.53. M.p. $197-198^{\circ} .{ }^{1} \mathrm{H}-\mathrm{NMR}\left(400 \mathrm{MHz}, \mathrm{CDCl}_{3}\right)$ : 0.90-1.03 (m, $\left.2 \mathrm{Me}\right) ; 1.08-1.24$ ( $m, 1 \mathrm{H}$ of $\left.\mathrm{CH}_{2}\right) ; 1.29(t, J=7.2, \mathrm{Me}) ; 1.38-1.59\left(m, 2^{t} \mathrm{Bu}, \mathrm{CH}_{2}\right) ; 1.83-1.95\left(m, 1 \mathrm{H}\right.$ of $\left.\mathrm{CH}_{2}\right) ; 1.97-2.21\left(m, \mathrm{CH}_{2}\right)$; 3.51-3.65 ( $m, \mathrm{H}-\mathrm{C}(1))$; 3.89-3.99 ( $m, \mathrm{CH})$; $4.02-4.73\left(m, \mathrm{CH}, \mathrm{CH}_{2} \mathrm{O}, \mathrm{H}-\mathrm{C}(2), \mathrm{H}-\mathrm{C}(3), \mathrm{H}-\mathrm{C}(4)\right)$. ${ }^{13} \mathrm{C}-\mathrm{NMR}\left(100 \mathrm{MHz}, \mathrm{CDCl}_{3}\right): 11.4 ; 11.5 ; 14.0 ; 21.2 ; 22.7 ; 27.9 ; 27.94 ; 35.0 ; 41.1 ; 46.1 ; 50.6 ; 51.9 ; 54.2 ; 50.2$; $71.7 ; 78.9 ; 79.8 ; 154.1 ; 157.2 ; 174.0$. ESI-MS: $495\left([M+\mathrm{Na}]^{+}\right)$. Anal. calc. for: $\mathrm{C}_{24} \mathrm{H}_{44} \mathrm{~N}_{2} \mathrm{O}_{7}: \mathrm{C} 60.99, \mathrm{H}$ 9.38, N 5.93; found: C 60.98, H 9.40, N 5.91 .

Ethyl (1R*,2S*,3R*,4S*)-2-\{[(tert-Butoxy)carbonyl]amino\}-4-((1S*)-1-\{[(tert-butoxy) carbonyl]aminol-2-ethylbutyl)-3-hydroxycyclopentanecarboxylate (22). Yellowish oil. Yield: $58 \% . R_{\mathrm{f}}$ (hexane/ AcOEt) 0.47. ${ }^{1} \mathrm{H}-\mathrm{NMR}\left(400 \mathrm{MHz}, \mathrm{CDCl}_{3}\right): 0.90-1.01(m, 2 \mathrm{Me}) ; 1.09-1.34\left(m, \mathrm{Me}, 2 \mathrm{CH}_{2}\right) ; 1.46(s$, $\left.2{ }^{t} \mathrm{Bu}\right) ; 1.92-2.30\left(m, \mathrm{CH}_{2}, \mathrm{H}-\mathrm{C}(1)\right) ; 2.76-2.88(m, \mathrm{H}-\mathrm{C}(4)) ; 2.94-3.02(m, \mathrm{CH}) ; 3.92-4.03(m, \mathrm{CH})$; $4.06-4.31\left(m, \mathrm{H}-\mathrm{C}(2), \mathrm{H}-\mathrm{C}(3), \mathrm{CH}_{2} \mathrm{O}\right) ; 4.35-4.44$ (br. $\left.s, \mathrm{NH}\right) ; 5.07-5.19$ (br. $\left.s, \mathrm{NH}\right) .{ }^{13} \mathrm{C}-\mathrm{NMR}$ $\left(100 \mathrm{MHz}, \mathrm{CDCl}_{3}\right): 11.0 ; 12.0 ; 14.3 ; 21.0 ; 24.3 ; 26.2 ; 27.0 ; 28.4 ; 44.3 ; 45.4 ; 48.0 ; 51.6 ; 58.8 ; 63.0 ; 73.8 ; 80.0$; 
127.0; 142.0; 153.7; 158.1. ESI-MS: $474\left([M+1]^{+}\right)$. Anal. calc. for $\mathrm{C}_{24} \mathrm{H}_{44} \mathrm{~N}_{2} \mathrm{O}_{7}: \mathrm{C} 60.99, \mathrm{H} 9.38, \mathrm{~N} 5.93$; found: C 60.97, H 9.39, N 5.94.

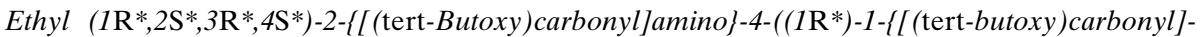
amino\}-2-ethylbutyl)-3-hydroxycyclopentanecarboxylate (23). White solid. Yield: $20 \% . R_{\mathrm{f}}$ (hexane/ AcOEt) 0.35. M.p. $137-138^{\circ} .{ }^{1} \mathrm{H}-\mathrm{NMR}\left(400 \mathrm{MHz}, \mathrm{CDCl}_{3}\right): 0.89-1.00(m, 2 \mathrm{Me}) ; 1.29(t, J=7.1, \mathrm{Me})$; $1.40-1.72\left(m, 2{ }^{t} \mathrm{Bu}, 4 \mathrm{CH}_{2}\right) ; 1.96-2.52\left(m, \mathrm{CH}_{2}, \mathrm{H}-\mathrm{C}(1), \mathrm{H}-\mathrm{C}(4), \mathrm{CH}\right) ; 3.60-3.76(m, \mathrm{CH}) ; 4.07-4.42$ $\left(m, \mathrm{H}-\mathrm{C}(2), \mathrm{CH}_{2} \mathrm{O}, \mathrm{H}-\mathrm{C}(3)\right) ; 4.84-4.93$ (br. $\left.s, \mathrm{NH}\right) ; 5.16-5.30$ (br. $\left.s, \mathrm{NH}\right) .{ }^{13} \mathrm{C}-\mathrm{NMR}(100 \mathrm{MHz}$, $\left.\mathrm{CDCl}_{3}\right): 11.5 ; 11.9 ; 13.7 ; 21.2 ; 22.6 ; 25.2 ; 27.9 ; 27.9 ; 44.3 ; 44.7 ; 46.3 ; 50.9 ; 58.8 ; 60.3 ; 74.2 ; 79.1 ; 126.9$; 142.0; 154.9; 156.3. ESI-MS: $474\left([M+1]^{+}\right)$. Anal. calc. for $\mathrm{C}_{24} \mathrm{H}_{44} \mathrm{~N}_{2} \mathrm{O}_{7}: \mathrm{C} 60.99, \mathrm{H} 9.38, \mathrm{~N} 5.93$; found: $\mathrm{C}$ 61.01, H 9.36, N 5.92.

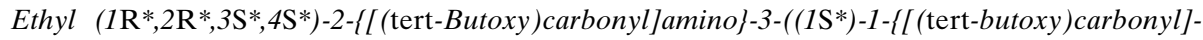
amino\}ethyl)-4-fluorocyclopentanecarboxylate (24a). White solid. Yield: 24\%. $R_{\mathrm{f}}$ (hexane/AcOEt) 0.56. M.p. $114-115^{\circ} .{ }^{1} \mathrm{H}-\mathrm{NMR}\left(400 \mathrm{MHz}, \mathrm{CDCl}_{3}\right): 1.23(d, J=6.9, \mathrm{Me}) ; 1.27-1.33(\mathrm{~m}, \mathrm{Me}) ; 1.43-1.49$ $\left(m, 2{ }^{\mathrm{t}} \mathrm{Bu}\right) ; 2.11-2.42\left(m, \mathrm{CH}_{2}, \mathrm{H}-\mathrm{C}(1)\right) ; 3.00-3.08(m, \mathrm{H}-\mathrm{C}(3)) ; 4.41-4.28\left(m, \mathrm{NH}, \mathrm{CH}, \mathrm{CH}_{2} \mathrm{O}\right) ; 4.83-$ $5.09(m, \mathrm{H}-\mathrm{C}(4), \mathrm{H}-\mathrm{C}(2)) ; 5.58-5.67$ (br. $s, \mathrm{NH}) .{ }^{13} \mathrm{C}-\mathrm{NMR}\left(100 \mathrm{MHz}, \mathrm{CDCl}_{3}\right)$ : $14.5 ; 19.6 ; 28.8 ; 34.8$; $34.9 ; 45.2 ; 46.1 ; 52.2 ; 55.9 ; 56.1 ; 61.3 ; 79.1 ; 79.8 ; 95.9 ; 152.8 ; 173.5$. ESI-MS: $419\left([M+1]^{+}\right)$. Anal. calc. for: $\mathrm{C}_{20} \mathrm{H}_{35} \mathrm{FN}_{2} \mathrm{O}_{6}$ : C 57.40, H 8.43, N 6.69; found: C 57.43, H 8.42, N 5.67.

Ethyl (1R*,2R*)-2-\{[(tert-butoxy)carbonyl]amino\}-3-((1S*)-1-\{[(tert-butoxy)carbonyl ]amino $\}$ ethyl)cyclopent-3-ene-1-carboxylate (25a). White solid. Yield: $45 \% . R_{\mathrm{f}}($ hexane/AcOEt) 0.52 . M.p. 83$84^{\circ} .{ }^{1} \mathrm{H}-\mathrm{NMR}\left(400 \mathrm{MHz}, \mathrm{CDCl}_{3}\right): 1.26-1.33(m, 2 \mathrm{Me}) ; 1.42-1.50\left(m, 2 \mathrm{Me}_{3} \mathrm{C}\right) ; 2.41-2.52(m, 1 \mathrm{H}$ of $\left.\mathrm{CH}_{2}\right) ; 2.75-2.86\left(m, 1 \mathrm{H}\right.$ of $\left.\mathrm{CH}_{2}\right) ; 3.36-3.45(m, \mathrm{H}-\mathrm{C}(1)) ; 4.12-4.22\left(m, \mathrm{CH}_{2} \mathrm{O}\right) ; 4.34$ (br. $\left.s, \mathrm{NH}\right) ; 4.70-$ $5.08(m, \mathrm{NH}, \mathrm{CH}, \mathrm{H}-\mathrm{C}(2)) ; 5.65(s, \mathrm{H}-\mathrm{C}(4)) .{ }^{13} \mathrm{C}-\mathrm{NMR}\left(100 \mathrm{MHz}, \mathrm{CDCl}_{3}\right): 14.6 ; 21.2 ; 28.7 ; 28.8 ; 33.3$; 45.7; 47.7; 57.4; 61.0; 79.9; 121.4; 126.2; 155.5; 158.5; 172.9. ESI-MS: $399\left([M+1]^{+}\right)$. Anal. calc. for $\mathrm{C}_{20} \mathrm{H}_{34} \mathrm{~N}_{2} \mathrm{O}_{6}$ : C 60.28, H 8.60, N 7.03; found: C 60.26, H 8.61, N 7.04.

Ethyl $\left(1 \mathrm{R}^{*}, 2 \mathrm{R} * 3 \mathrm{~S}^{*}, 4 \mathrm{~S}^{*}\right)-2-\left\{\left[(\right.\right.$ tert-Butoxy) carbonyl]amino $\}-3-\left(\left(1 \mathrm{~S}^{*}\right)-1-\{[\right.$ (tert-butoxy) carbonyl]amino\}propyl)-4-fluorocyclopentanecarboxylate $(\mathbf{2 4 b})$. White solid. Yield: $30 \% . R_{\mathrm{f}}$ (hexane/AcOEt) 0.74. M.p. $103-104^{\circ} .{ }^{1} \mathrm{H}-\mathrm{NMR}\left(400 \mathrm{MHz}, \mathrm{CDCl}_{3}\right): 0.97(t, J=7.3, \mathrm{Me}) ; 1.26-1.33(\mathrm{~m}, \mathrm{Me}) ; 1.44-1.50$ $\left(m, 2{ }^{t} \mathrm{Bu}\right) ; 1.57-1.67\left(m, \mathrm{CH}_{2}\right) ; 2.09-2.45\left(m, \mathrm{CH}_{2}, \mathrm{H}-\mathrm{C}(1)\right) ; 2.97-3.06(m, \mathrm{H}-\mathrm{C}(3)) ; 3.86$ (br. $\left.s, \mathrm{NH}\right)$; $4.03-4.28\left(m, \mathrm{CH}_{2} \mathrm{O}, \mathrm{H}-\mathrm{C}(2)\right) ; 4.80-5.03(m, \mathrm{CH}, \mathrm{H}-\mathrm{C}(4)) ; 5.66-5.76$ (br. $\left.s, \mathrm{NH}\right) .{ }^{13} \mathrm{C}-\mathrm{NMR}(100 \mathrm{MHz}$, $\left.\mathrm{CDCl}_{3}\right): 10.5 ; 13.6 ; 26.4 ; 27.9 ; 34.1 ; 44.5 ; 50.9 ; 51.3 ; 54.0 ; 60.5 ; 78.8 ; 79.3 ; 94.5 ; 95.9 ; 155.0 ; 155.9 ; 173.3$. ESI-MS: $434\left([M+1]^{+}\right)$. Anal. calc. for $\mathrm{C}_{21} \mathrm{H}_{37} \mathrm{FN}_{2} \mathrm{O}_{6}$ : C 58.31, $\mathrm{H} 8.62, \mathrm{~N} 6.48$; found: $\mathrm{C} 58.29, \mathrm{H} 8.63, \mathrm{~N}$ 6.49 .

Ethyl $\left(1 \mathrm{R}^{*}, 2 \mathrm{R} *\right)-2-\{[($ tert-Butoxy) carbonyl]amino\}-3-((1S*)-1-\{[(tert-butoxy)carbonyl]amino\}propyl)cyclopent-3-ene-1-carboxylate (25b). Brownish oil. Yield: 43\%. $R_{\mathrm{f}}$ (hexane/AcOEt) 0.67. ${ }^{1} \mathrm{H}-\mathrm{NMR}$ $\left(400 \mathrm{MHz}, \mathrm{CDCl}_{3}\right): 0.91(t, J=7.5, \mathrm{Me}) ; 1.29(t, J=7.2, \mathrm{Me}) ; 1.42-1.50\left(m,{ }^{t} \mathrm{Bu}\right) ; 1.62-1.77\left(m, \mathrm{CH}_{2}\right)$; $2.34-2.53\left(m, 1 \mathrm{H}\right.$ of $\left.\mathrm{CH}_{2}\right) ; 2.74-2.90\left(m, 1 \mathrm{H}\right.$ of $\left.\mathrm{CH}_{2}\right)$; 3.30-3.45 $(m, \mathrm{H}-\mathrm{C}(1)) ; 4.09-4.28(m, \mathrm{CH}$, $\left.\mathrm{CH}_{2} \mathrm{O}\right) ; 4.78-5.05(m, 2 \mathrm{NH}, \mathrm{H}-\mathrm{C}(2)) ; 5.65(s, \mathrm{H}-\mathrm{C}(4)) .{ }^{13} \mathrm{C}-\mathrm{NMR}\left(100 \mathrm{MHz}, \mathrm{CDCl}_{3}\right)$ : 9.6; 13.8; 26.6; $27.9 ; 27.9 ; 32.5 ; 46.7 ; 56.6 ; 60.2 ; 79.1 ; 112.5 ; 115.4 ; 117.2 ; 126.4 ; 154.8 ; 171.3 ; 172.2$. ESI-MS: $414\left([M+1]^{+}\right)$. Anal. calc. for $\mathrm{C}_{21} \mathrm{H}_{36} \mathrm{~N}_{2} \mathrm{O}_{6}$ : C 61.14, H 8.80, N 6.79; found: C 61.13, H 8.82, N 6.80.

Ethyl $\left(1 \mathrm{R}^{*}, 2 \mathrm{R}^{*}, 3 \mathrm{~S}^{*}, 4 \mathrm{~S}^{*}\right)-2-\left\{[(\right.$ tert-Butoxy $)$ carbonyl]amino $\}-3-\left(\left(1 \mathrm{~S}^{*}\right)-1-\{[(\right.$ tert-butoxy $)$ carbonyl $]-$ amino\}-2-ethylbutyl)-4-fluorocyclopentanecarboxylate $\mathbf{( 2 4 c )}$. White solid. Yield: $28 \% . R_{\mathrm{f}}$ (hexane/ AcOEt) 0.75. M.p. $115-116^{\circ} .{ }^{1} \mathrm{H}-\mathrm{NMR}\left(400 \mathrm{MHz}, \mathrm{CDCl}_{3}\right): 0.83-0.95$ ( $\left.m, 2 \mathrm{Me}\right) ; 1.17-1.37$ ( $m, \mathrm{Me}$, $\left.\mathrm{CH}_{2}\right) ; 1.40-1.52\left(m, 2{ }^{\mathrm{t}} \mathrm{Bu}, \mathrm{CH}_{2}\right) ; 2.10-2.38\left(m, \mathrm{CH}_{2}, \mathrm{CH}\right) ; 2.46-2.62(m, \mathrm{H}-\mathrm{C}(1)) ; 2.98-3.09(m$, $\mathrm{H}-\mathrm{C}(3)) ; 3.79-3.90(m, \mathrm{CH}) ; 4.03-4.29\left(m, \mathrm{H}-\mathrm{C}(2), \mathrm{CH}_{2} \mathrm{O}\right) ; 4.82($ br. $s, \mathrm{NH}) ; 4.91-5.06(m, \mathrm{H}-\mathrm{C}(4))$; 5.68-5.79 (br. $s, \mathrm{NH}) .{ }^{13} \mathrm{C}-\mathrm{NMR}\left(100 \mathrm{MHz}, \mathrm{CDCl}_{3}\right): 9.5 ; 10.3 ; 13.6 ; 20.8 ; 21.4 ; 27.9 ; 34.0 ; 41.9 ; 44.7 ; 51.1$; $51.2 ; 51.5 ; 60.5 ; 78.7 ; 79.2 ; 95.3 ; 96.7 ; 154.9 ; 156.0 ; 173.4$. ESI-MS: $475\left([M+1]^{+}\right)$. Anal. calc. for $\mathrm{C}_{24} \mathrm{H}_{43} \mathrm{FN}_{2} \mathrm{O}_{6}$ : C 60.74, H 9.13, N 5.90; found: C 60.73, H 9.12, N 5.92.

Ethyl $\left(1 \mathrm{R}^{*}, 2 \mathrm{R}^{*}\right)-2-\left\{\left[(\right.\right.$ tert-Butoxy) carbonyl]amino $\}-3-\left(\left(1 \mathrm{~S}^{*}\right)-1-\{[(\right.$ tert-butoxy $)$ carbonyl]amino $\}-2-$ ethylbutyl)cyclopent-3-ene-1-carboxylate (25c). White solid. Yield: $27 \% . R_{\mathrm{f}}$ (hexane/AcOEt) 0.61. M.p. 94-95 ${ }^{1}{ }^{1} \mathrm{H}-\mathrm{NMR}\left(400 \mathrm{MHz}, \mathrm{CDCl}_{3}\right)$ : 0.84-1.11 ( $\left.m, 2 \mathrm{Me}, \mathrm{CH}_{2}\right) ; 1.17-1.36\left(m, \mathrm{Me}, \mathrm{CH}_{2}\right) ; 1.39-1.53(m$, $\left.2{ }^{t} \mathrm{Bu}\right) ; 2.40-2.53\left(\mathrm{~m}, 1 \mathrm{H}\right.$ of $\left.\mathrm{CH}_{2}\right) ; 2.76-2.89\left(m, 1 \mathrm{H}\right.$ of $\left.\mathrm{CH}_{2}\right) ; 3.36-3.47(m, \mathrm{CH}) ; 4.09-4.25(m$, $\left.\mathrm{CH}_{2} \mathrm{O}\right) ; 436$ (br. $\left.s, \mathrm{NH}\right) ; 4.62-5.07(m, \mathrm{CH}, \mathrm{NH}, \mathrm{H}-\mathrm{C}(2)) ; 5.63-5.65(m, \mathrm{H}-\mathrm{C}(4)) .{ }^{13} \mathrm{C}-\mathrm{NMR}(100 \mathrm{MHz}$, $\left.\mathrm{CDCl}_{3}\right): 11.2 ; 12.2 ; 13.8 ; 21.1 ; 22.2 ; 27.8 ; 27.9 ; 32.4 ; 42.4 ; 46.7 ; 50.3 ; 56.5 ; 60.1 ; 78.8 ; 78.9 ; 126.5 ; 143.1$; 
154.6; 155.2; 172.1. ESI-MS: $455\left([M+1]^{+}\right)$. Anal. calc. for $\mathrm{C}_{24} \mathrm{H}_{42} \mathrm{~N}_{2} \mathrm{O}_{6}: \mathrm{C} 63.41, \mathrm{H}$ 9.31, N 6.16; found: $\mathrm{C}$ 63.39, H 9.32, N 6.17.

Ethyl (1R*,2S*,3S*,4S*)-2-\{[(tert-Butoxy) carbonyl]amino\}-4-((1S*)-1-\{[(tert-butoxy)carbonyl]amino\}ethyl)-3-fluorocyclopentanecarboxylate (26a). White solid. Yield: $26 \% . R_{\mathrm{f}}$ (hexane/AcOEt) 0.51. M.p. $104-105^{\circ} .{ }^{1} \mathrm{H}-\mathrm{NMR}\left(400 \mathrm{MHz}, \mathrm{CDCl}_{3}\right): 1.17-1.23(\mathrm{~m}, \mathrm{Me}) ; 1.29(t, J=7.2, \mathrm{Me}) ; 1.44-1.49(\mathrm{~m}$, $\left.2{ }^{t} \mathrm{Bu}\right) ; 2.09-2.24\left(m, 1 \mathrm{H}\right.$ of $\left.\mathrm{CH}_{2}\right) ; 2.27-2.58\left(m, 1 \mathrm{H}\right.$ of $\left.\mathrm{CH}_{2}, \mathrm{H}-\mathrm{C}(1)\right) ; 2.69-2.94(m, \mathrm{H}-\mathrm{C}(4)) ; 3.69-$ $3.89(m, \mathrm{CH}) ; 4.09-4.25\left(m, \mathrm{CH}_{2} \mathrm{O}, \mathrm{H}-\mathrm{C}(2)\right) ; 4.26-4.43$ (br. $\left.s, \mathrm{NH}\right) ; 4.65-5.00(m, \mathrm{NH}, \mathrm{H}-\mathrm{C}(3))$. ${ }^{13} \mathrm{C}-\mathrm{NMR}\left(100 \mathrm{MHz}, \mathrm{CDCl}_{3}\right): 13.7 ; 15.5 ; 27.9 ; 33.5 ; 36.6 ; 46.5 ; 48.1 ; 50.8 ; 51.5 ; 51.7 ; 60.6 ; 79.2 ; 103.6$; 105.0; 154.8; 172.6. ESI-MS: $419\left([M+1]^{+}\right)$. Anal. calc. for $\mathrm{C}_{20} \mathrm{H}_{35} \mathrm{FN}_{2} \mathrm{O}_{6}$ : C 57.40, $\mathrm{H} 8.43$, N 6.69; found: C 57.41, H 8.41, N 6.70.

Ethyl (1R*,4R*)-2-\{[(tert-Butoxy) carbonyl]amino\}-4-((1S*)-1-\{[(tert-butoxy)carbonyl]amino\}ethyl)cyclopent-2-ene-1-carboxylate (27a). White solid. Yield: $48 \% . R_{\mathrm{f}}$ (hexane/AcOEt) 0.47. M.p. 129-130 ${ }^{\circ}{ }^{1} \mathrm{H}-\mathrm{NMR}\left(400 \mathrm{MHz}, \mathrm{CDCl}_{3}\right): 1.18-1.3(\mathrm{~m}, \mathrm{Me}) ; 1.26-1.33(\mathrm{~m}, \mathrm{Me}) ; 1.45-1.48\left(\mathrm{~m},{ }^{t} \mathrm{Bu}\right)$; 2.29-2.49 ( $m, \mathrm{H}-\mathrm{C}(1)) ; 2.57-2.79\left(m, \mathrm{CH}_{2}\right) ; 2.81-2.91(m, \mathrm{H}-\mathrm{C}(4)) ; 3.70-3.87(m, \mathrm{NH}) ; 4.15-4.25(m$, $\left.\mathrm{CH}_{2} \mathrm{O}, \mathrm{CH}\right) ; 4.92-5.01(m, \mathrm{NH}) ; 5.45-5.47(m, \mathrm{H}-\mathrm{C}(3)) .{ }^{13} \mathrm{C}-\mathrm{NMR}\left(100 \mathrm{MHz}, \mathrm{CDCl}_{3}\right): 13.7 ; 19.4 ; 27.9$; 36.77; 44.9; 46.7; 46.6; 50.7; 60.4; 78.0; 79.2; 124.7; 148.3; 154.7; 167.8. ESI-MS: $399\left([M+1]^{+}\right)$. Anal. calc. for $\mathrm{C}_{20} \mathrm{H}_{34} \mathrm{~N}_{2} \mathrm{O}_{6}$ : C 60.28, $\mathrm{H} 8.60, \mathrm{~N} 7.03$; found: C 60.30, H 8.59, N 7.02.

Ethyl $\left(1 \mathrm{R}^{*}, 2 \mathrm{~S} *, 3 \mathrm{~S} *, 4 \mathrm{~S} *\right)-2-\{[$ (tert-Butoxy) carbonyl]amino $\}-4-((1 \mathrm{~S} *)-1-\{[($ tert-butoxy $)$ carbonyl]amino\}propyl)-3-fluorocyclopentanecarboxylate (26b). Brownish oil. Yield: $15 \% . R_{\mathrm{f}}$ (hexane/AcOEt) 0.57. ${ }^{1} \mathrm{H}-\mathrm{NMR}\left(400 \mathrm{MHz}, \mathrm{CDCl}_{3}\right)$ : $0.89-1.02(\mathrm{~m}, \mathrm{Me}) ; 1.25-1.33(\mathrm{~m}, \mathrm{Me}) ; 1.44-1.50\left(\mathrm{~m}, 2{ }^{t} \mathrm{Bu}\right) ; 1.64-$ $1.80\left(m, \mathrm{CH}_{2}\right) ; 2.23-3.06\left(m, \mathrm{CH}_{2}, \mathrm{H}-\mathrm{C}(1), \mathrm{CH}\right) ; 3.49-3.72(m, \mathrm{H}-\mathrm{C}(4)) ; 4.14-4.25\left(m, \mathrm{CH}_{2} \mathrm{O}\right) ; 4.29-$ $4.54(m, \mathrm{H}-\mathrm{C}(2)) ; 4.57-4.82(m, \mathrm{H}-\mathrm{C}(3)) ; 4.97$ (br. $s, \mathrm{NH}) ; 5.13$ (br. $s, \mathrm{NH}) .{ }^{13} \mathrm{C}-\mathrm{NMR}(100 \mathrm{MHz}$, $\left.\mathrm{CDCl}_{3}\right): 9.9 ; 13.7 ; 22.7 ; 27.9 ; 36.8 ; 47.9 ; 49.7 ; 52.9 ; 53.7 ; 57.3 ; 57.5 ; 60.5 ; 79.1 ; 103.8 ; 105.2 ; 154.3 ; 155.8$. ESI-MS: $433\left([M+1]^{+}\right)$. Anal. calc. for $\mathrm{C}_{21} \mathrm{H}_{37} \mathrm{FN}_{2} \mathrm{O}_{6}$ : C 58.31, $\mathrm{H}$ 8.62, $\mathrm{N} 6.48$; found: $\mathrm{C} 58.30, \mathrm{H}$ 8.61, $\mathrm{N}$ 6.50 .

Ethyl $(1 \mathrm{R} * 4 \mathrm{R} *)-2-\{[($ tert-Butoxy) carbonyl]amino $\}-4-((1 \mathrm{~S} *)-1-\{[($ tert-butoxy $)$ carbonyl]amino\}propyl)cyclopent-2-ene-1-carboxylate (27b). White solid. Yield: 39\%. $R_{\mathrm{f}}$ (hexane/AcOEt) 0.48. M.p. $121-$ $122^{\circ} .{ }^{1} \mathrm{H}-\mathrm{NMR}\left(400 \mathrm{MHz}, \mathrm{CDCl}_{3}\right): 0.88-1.01(\mathrm{~m}, \mathrm{Me}) ; 1.24-1.33(\mathrm{~m}, \mathrm{Me}) ; 1.47\left(\mathrm{~s}, 2{ }^{t} \mathrm{Bu}\right) ; 1.61-1.90(\mathrm{~m}$, $\left.\mathrm{CH}_{2}\right) ; 2.09-2.35\left(m, 1 \mathrm{H}\right.$ of $\left.\mathrm{CH}_{2}\right) ; 2.53-2.75\left(m, 1 \mathrm{H}\right.$ of $\left.\mathrm{CH}_{2}\right) ; 2.77-2.92(m, \mathrm{H}-\mathrm{C}(1)) ; 4.07-4.25(m$, $\left.\mathrm{H}-\mathrm{C}(4), \mathrm{CH}_{2} \mathrm{O}\right) ; 4.47$ (br. $\left.s, \mathrm{NH}\right) ; 4.56-4.87(m, \mathrm{CH}) ; 4.93-5.05(m, \mathrm{NH}) ; 5.45-5.47(m, \mathrm{H}-\mathrm{C}(3))$. ${ }^{13} \mathrm{C}-\mathrm{NMR}\left(100 \mathrm{MHz}, \mathrm{CDCl}_{3}\right): 9.8 ; 13.7 ; 22.6 ; 26.1 ; 27.9 ; 50.6 ; 51.5 ; 57.3 ; 60.5 ; 79.0 ; 103.8 ; 105.2 ; 124.6$; 154.6; 155.8; 172.7. ESI-MS: $413\left([M+1]^{+}\right)$. Anal. calc. for $\mathrm{C}_{21} \mathrm{H}_{36} \mathrm{~N}_{2} \mathrm{O}_{6}: \mathrm{C} 61.14, \mathrm{H}$ 8.80, N 6.79; found: C $61.15, \mathrm{H} 8.78, \mathrm{~N} 6.80$.

Ethyl $\left(1 \mathrm{R}^{*}, 4 \mathrm{R}^{*}\right)-2-\left\{\left[(\right.\right.$ tert-Butoxy) carbonyl]amino $\}-4-\left(\left(1 \mathrm{~S}^{*}\right)-1-\{[(\right.$ tert-butoxy $)$ carbonyl]amino $\}-2-$ ethylbutyl)cyclopent-2-ene-1-carboxylate $(\mathbf{2 7 c})$. Yellowish oil. Yield: $58 \% . R_{\mathrm{f}}$ (hexane/AcOEt) 0.48 . ${ }^{1} \mathrm{H}-\mathrm{NMR}\left(400 \mathrm{MHz}, \mathrm{CDCl}_{3}\right)$ : 0.85-1.17 (m, $\left.2 \mathrm{Me}, \mathrm{CH}_{2}\right)$; $1.23-1.39\left(m, \mathrm{Me}, \mathrm{CH}_{2}\right) ; 1.46\left(s, 2{ }^{\mathrm{t}} \mathrm{Bu}\right) ; 1.58-$ $1.68\left(m, \mathrm{CH}_{2}\right) ; 2.41-2.90(m, \mathrm{CH}, \mathrm{H}-\mathrm{C}(1), \mathrm{CH}) ; 3.51-3.61(m, \mathrm{H}-\mathrm{C}(4)) ; 4.07-4.22\left(m, \mathrm{CH}_{2} \mathrm{O}\right) ; 4.37$ (br. $s, \mathrm{NH}) ; 4.49$ (br. $s, \mathrm{NH}) ; 5.63-5.64(m, \mathrm{H}-\mathrm{C}(3)) .{ }^{13} \mathrm{C}-\mathrm{NMR}\left(100 \mathrm{MHz}, \mathrm{CDCl}_{3}\right): 10.8 ; 11.2 ; 13.7 ; 21.5$; $22.6 ; 27.8 ; 27.9 ; 34.4 ; 43.1 ; 50.2 ; 52.0 ; 57.11 ; 60.4 ; 78.5 ; 79.1 ; 103.2 ; 105.5 ; 153.9 ; 155.8 ; 171.7$. ESI-MS: 455 $\left([M+1]^{+}\right)$. Anal. calc. for $\mathrm{C}_{24} \mathrm{H}_{42} \mathrm{~N}_{2} \mathrm{O}_{6}$ : C 63.41, H 9.31, N 6.16; found: C 63.44, H 9.30, N 6.14.

$X$-Rray Crystallographic Studies. Crystallographic data for the compounds $\mathbf{1 1}$ and $\mathbf{2 0}$ were collected with Agilent Supernova diffractometer equipped with Atlas area detector using $\mathrm{Cu} K_{\alpha}$ radiation $(\lambda=$ $1.54184 \AA$ ). Empirical absorption correction, using spherical harmonics implemented in SCALE3 ABSPACK scaling algorithm, was applied for both compounds with CrysAlisPro program package [9]. The structures were solved by direct methods using SIR97 [10] program, and full-matrix, least-squares refinements on $F^{2}$ were performed using the SHELXL-97 [11] program. Molecular structure figures were drawn with Diamond3 program [12]. Selected crystallographic data collected in CCDC-902350 and -902351 contain the supplementary crystallographic data for $\mathbf{1 1}$ and $\mathbf{2 0}$. These data can be obtained free of charge via ttp://www.ccdc.cam.ac.uk/conts/retrieving.html, or from the Cambridge Crystallographic Data Centre, 12 Union Road, Cambridge CB21EZ, UK; fax: (+44)1223-336-033; or e-mail: deposit@ccdc. cam.ac.uk. 


\section{REFERENCES}

[1] a) F. Fülöp, Chem. Rev. 2001, 101, 2181; b) L. Kiss, E. Forró, F. Fülöp, 'Synthesis of carbocyclic $\beta$ amino acids', in 'Amino Acids, Peptides and Proteins in Organic Chemistry', Vol. 1, Ed. A. B. Hughes, Wiley, Weinheim, 2009, p. 367; c) L. Kiss, F. Fülöp, Synlett 2010, 1302; d) D. Seebach, A. K. Beck, S. Capone, D. Deniau, U. Grošelj, E. Zass, Synthesis 2009, 1; e) S. Abele, D. Seebach, Eur. J. Org. Chem. 2000, 1; f) D. J. Ramón, G. Guilenna, D. Seebach, Helv. Chim. Acta 1996, 79, 875; g) Z. Hameršak, M. Roje, A. Avdagić, V. Šunjić, Tetrahedron: Asymmetry 2007, 18, 635; h) S. K. Pandey, G. F. Jogdand, J. C. A. Oliveira, R. A. Mata, P. R. Rajamohanan, C. V. Ramana, Chem.-Eur. J. 2011, 17, 12946.

[2] T. A. Martinek, F. Fülöp, Eur. J. Biochem. 2003, 270, 3657; E. Torres, C. Acosta-Silva, F. Rúa, Á. Álvarez-Larena, T. Parella, V. Branchadell, R. M. Ortuño, Tetrahedron 2009, 65, 5669; D. Fernández, E. Torres, F. X. Avilés, R. M. Ortuño, J. Vendrell, Bioorg. Med. Chem. 2009, 17, 3824; C. Fernandes, E. Pereira, S. Faure, D. J. Aitken, J. Org. Chem. 2009, 74, 3217; O. Roy, S. Faure, D. J. Aitken, Tetrahedron Lett. 2006, 47, 5981; F. Rúa, S. Boussert, T. Parella, I. Díez-Pérez, V. Branchadell, E. Giralt, R. M. Ortuño, Org. Lett. 2007, 9, 3643; V. D’Elia, H. Zwicknagl, O. Reiser, J. Org. Chem. 2008, 73, 3262; C. Fernandes, S. Faure, E. Pereira, V. Théry, V. Declerck, R. Guillot, D. J. Aitken, Org. Lett. 2010, 12, 3606; F. Fülöp, T. A. Martinek, G. K. Tóth, Chem. Soc. Rev. 2006, 35, 323; S. Celis, E. Gorrea, P. Nolis, O. Illa, R. M. Ortuño, Org. Biomol. Chem. 2012, 10, 861; T. A. Martinek, F. Fülöp, Chem. Soc. Rev. 2012, 41, 687; E. Gorrea, P. Nolis, E. Torres, E. Da Silva, D. B. Amabilino, V. Branchadell, R. M. Ortuño, Chem.-Eur. J. 2011, 17, 4588.

[3] A. J. Oakley, S. Barrett, T. S. Peat, J. Newman, V. A. Streltsov, L. Waddington, T. Saito, M. Tashiro, J. L. McKimm-Breschkin, J. Med. Chem. 2010, 53, 6421; W. J. Lü, Y. L. Chen, W. P. Ma, X. Y. Zhang, F. Luan, M. C. Liu, X. G. Chen, Z. D. Hu, Eur. J. Med. Chem. 2008, 43, 569; P. Chand, P. L. Kotian, A. Dehghani, Y. El-Kattan, T.-H. Lin, T. L. Hutchison, Y. S. Babu, S. Bantia, A. J. Elliott, J. A. Montgomery, J. Med. Chem. 2001, 44, 4379; P. Chand, Y. S. Babu, S. Bantia, S. Rowland, A. Dehghani, P. L. Kotian, T. L. Hutchison, S. Ali, W. Brouillette, Y. El-Kattan, T.-H. Lin, J. Med. Chem. 2004, 47, 1919; X. Yi, Z. Guo, F. M. Chu, Bioorg. Med. Chem. 2003, 11, 1465; P. Chand, S. Bantia, P. L. Kotian, Y. El-Kattan, T.-H. S. Lin, Y. S. Babu, Bioorg. Med. Chem. 2005, 13, 4071; Y. Cui, Z. Jiao, J. Gong, Q. Yu, X. Zheng, J. Quan, M. Luo, Z. Yang, Org. Lett. 2010, 12, 4; X. Yi, Z. Guo, F. M. Chu, Bioorg. Med. Chem. 2003, 11, 1465.

[4] N. C. Yoder, K. Kumar, Chem. Soc. Rev. 2002, 31, 335; R. I. Mathad, B. Jaun, O. Flögel, J. Gardiner, M. Löweneck, J. D. C. Codée, P. H. Seeberger, D. Seebach, M. K. Edmonds, F. H. M. Graichen, A. D. Abell, Helv. Chim. Acta 2007, 90, 2251; X.-L.Qiu, W.-D. Meng, F.-L. Qing, Tetrahedron 2004, 60, 6711; A. Sutherland, C. L. Willis, Nat. Prod. Rep. 2000, 17, 621; R. Smits, C. D. Cadicamo, K. Burger, B. Koksch, Chem. Soc. Rev. 2008, 37, 1727; A. E. Sorochinsky, V. A. Soloshonok, J. Fluorine Chem. 2010, 131, 127; Y. Pan, Y. Zhao, T. Ma, Y. Yang, H. Liu, Z. Jiang, C.-H. Tan, Chem. -Eur. J. 2010, 16, 779; S. Capone, I. Kieltsch, O. Flögel, G. Lelais, A. Togni, D. Seebach, Helv. Chim. Acta 2008, 91, 2035; D. F. Hook, F. Gessier, C. Noti, P. Kast, D. Seebach, ChemBioChem 2004, 5, 691; C. Jäckel, W. Seufert, S. Thust, B. Koksch, ChemBioChem 2004, 5, 717; J. L. Aceña, A. E. Sorochinsky, V. A. Soloshonok, Synthesis 2012, 44, 1591; M. Salwiczek, E. K. Nyakatura, U. I. M. Gerling, S. Ye, B. Koksch, Chem. Soc. Rev. 2012, 41, 2135; V. S. Yarmolchuk, P. K. Mykhailiuk, I. V. Komarov, Tetrahedron Lett. 2011, 52, 1300; I. Yamamoto, M. J. T. Jordan, N. Gavande, M. R. Doddareddy, M. Chebib, L. Hunter, Chem. Commun. 2012, 48, 829.

[5] J. Luis Acena, A. Simon-Fuentes, S. Fustero, Curr. Org. Chem. 2010, 14, 928; S. Fustero, J. F. SanzCervera, J. L. Acena, M. Sanchez-Rosello, Synlett 2009, 525; K. Mikami, S. Fustero, M. SánchezRoselló, J. L. Aceña, V. Soloshonok, A. Sorochinsky, Synthesis 2011, 3045.

[6] a) L. Kiss, E. Forró, S. Fustero, F. Fülöp, Eur. J. Org. Chem. 2011, 4993; b) L. Kiss, E. Forró, S. Fustero, F. Fülöp, Org. Biomol. Chem. 2011, 9, 6528 c) J. Mittendorf, F. Kunisch, M. Matzke, H.-C. Militzer, A. Schmidt, W. Schönfeld, Bioorg. Med. Chem. Lett. 2003, 13, 433; d) S. Fustero, J. F. SanzCervera, J. Piera, M. Sánchez-Roselló, G. Chiva, A. Simón-Fuentes, J. Fluorine Chem. 2004, 125, 621 ; e) S. Fustero, M. Sánchez-Roselló, J. L. Aceña, B. Fernandez, A. Asensio, J. F. Sanz-Cervera, C. 
del Pozo, J. Org. Chem. 2009, 74, 3414; f) S. Fustero, M. Sánchez-Roselló, J. F. Sanz-Cervera, J. L. Aceña, C. del Pozo, B. Fernández, A. Bartolomé, A. Asensio, Org. Lett. 2006, 8, 4633.

[7] M. Nonn, L. Kiss, R. Sillanpää, F. Fülöp, Beilstein J. Org. Chem. 2012, 8, 100.

[8] a) L. Kiss, M. Nonn, E. Forró, R. Sillanpää, F. Fülöp, Tetrahedron Lett. 2009, 50, 2605; b) M. Nonn, L. Kiss, E. Forró, Z. Mucsi, F. Fülöp, Tetrahedron 2011, 67, 4079.

[9] R. C. Clark, J. S. Reid, Acta Cryst., Sect. A 1995, 51, 887; CrysAlis PRO, 2012, Agilent Technologies, Yarnton, England.

[10] A. Altomare, M. C. Burla, M. Camalli, G. L. Cascarano, C. Giacovazzo, A. Guagliardi, A. G. G. Moliterni, G. Polidori, R. Spagna, J. Appl. Crystallogr. 1999, 32, 115.

[11] G. M. Sheldrick, Acta Crystallogr., Sect. A 2008, 64, 112.

[12] Diamond - Crystal and Molecular Structure Visualization, Crystal Impact, K. Brandenburg and H. Putz, Postfach 1251, D-53002 Bonn. 